\title{
Scanning Tunneling Microscopy and Spectroscopy of Supported Nanostructures
}

\author{
Wolf-Dieter Schneider*
}

\begin{abstract}
Recent advances in low-temperature scanning tunneling microscopy (STM) and spectroscopy (STS) have provided new opportunities for the investigation of the local geometric, electronic, magnetic, and optical properties of nanostructures. This review focuses on the presentation and discussion of single molecules, supramolecular assemblies, and other nanostructures; all research results obtained in our laboratory. The emphasis is directed to the observation of new effects, where the properties of matter at the nanoscale differ from those at the mesoscopic or macroscopic scale: small is different. This fact is illustrated for the conservation of chirality in a hierarchical supramolecular assembly of organic molecules and for local light emission from supported molecules. The latter indicates a possible route towards an optical spectroscopic analysis on the scale of single molecules.
\end{abstract}

Keywords: Scanning tunneling microscopy · Single molecule luminescence $\cdot$ Supramolecular self-assembly . Surface chirality

\section{Introduction}

The quest for a reliable method for fabricating ordered atomic-scale structures is a prerequisite for future atomic-scale technology. The interest in such nanostructured materials, consisting of building blocks of a small number of atoms or molecules, arises from their promising new optical, catalytic, magnetic and electronic properties, which are fundamentally different from their macroscopic bulk counterparts. A promising route leading towards the fabrication of ordered nanoscale structures is based on self-assembly of basic building blocks of matter, i.e. of atoms and/or molecules. ${ }^{[1]}$ The key challenges are on one hand, to observe and to characterize the atomic and molecular processes leading to self-assembly and, on the other hand, to determine the electronic and optical properties as well as the chemical identity of the molecular building blocks and of the resulting nanostructures on the molecular level. Here we address two aspects of this challenge employing low-temperature scanning probe techniques. We discuss

${ }^{\star}$ Correspondence: Prof. Dr. W.-D. Schneider Ecole Polytechnique Fédérale de Lausanne (EPFL) Institut de Physique de la Matière Condensée $\mathrm{CH}-1015$ Lausanne

Tel.: +41216930444

Fax: +41216930422

E-mail: wolf-dieter.schneider@epfl.ch the chirality and supramolecular self-assembly of two organic molecules on the reconstructed $\mathrm{Au}(111)$ surface. The confinement of 1-nitronaphthalene (NN) to two dimensions introduces chirality not present in the gas phase, ${ }^{[2-7]}$ and chirality is conserved in a hierarchical supramolecular self-assembly of pentagonal symmetry of the organic molecule rubrene. We show the spontaneous chiral resolution of the racemate into disjointed homochiral complex architectures and demonstrate the ability to monitor directly the evolution of chiral recognition processes on the molecular and supramolecular level. ${ }^{[8-13]}$ In the second example, taking advantage of inelastic electron tunneling processes, we report on the excitation of luminescence from $\mathrm{C}_{60}$ and $\mathrm{C}_{70}$ molecules in the surface layer of fullerene nanocrystals self-assembled on an ultrathin $\mathrm{NaCl}$ film on $\mathrm{Au}(111)$. The observed fluorescence and phosphorescence spectra are found to be characteristic for the two molecular species, leading to unambiguous chemical recognition on the molecular scale. ${ }^{[14-16]}$

\section{Experimental}

The experiments were performed with a home-built low-temperature ultrahigh vacuum (UHV) STM operating at a pressure of $10^{-11}$ mbar and a temperature of 5 K or $50 \mathrm{~K}$, using cut PtIr or etched W tips. The rubrene molecules were deposited at low coverage $(0.3 \mathrm{ML})$ in situ by sublimation from a home-built evaporator onto a clean $\mathrm{Au}(111)$ substrate at room tempera- ture. $\mathrm{C}_{60}$ and $\mathrm{C}_{70}$ nanocrystals were grown on thin insulating $\mathrm{NaCl}$ layers deposited onto an atomically flat $\mathrm{Au}(111)$ substrate. $\mathrm{NaCl}$ was deposited from a resistively heated evaporator onto a clean $\mathrm{Au}(111)$ surface at room temperature. Subsequently, the fullerenes were sublimated onto the $\mathrm{NaCl}$-covered substrate forming fullerene nanocrystals of hexagonal and truncated triangular shape. Experiments were performed at a temperature of $50 \mathrm{~K}$. Photons emitted from the tunnel junction were collected by a plano-convex lens $(\mathrm{NA}=0.34)$ near the tip-sample gap along the direction $60^{\circ}$ with respect to the surface normal. The collected beam was then transmitted through a view port outside the UHV chamber and guided simultaneously to (i) a grating spectrometer $(50 \mathrm{l} / \mathrm{mm})$ coupled to a liquid-nitrogen-cooled CCD camera for spectral analysis ( $90 \%$ of the signal) and to (ii) an avalanche photodiode to record the total light intensity and to optimize the alignment of the lens with the tunnel junction (10\% of the signal). For the light emission measurements, the tip was positioned over a target location with a fixed tunnel resistance. Spectra were not corrected for the wavelength dependent sensitivity of the detection system. The wavelength resolution of the experiment was $8 \mathrm{~nm}$, corresponding to $\approx 20 \mathrm{meV}$ in the energy range of interest. The spectra were acquired with closed feedback loop while tunneling over a defined position on the sample, e.g. over a single molecule, with a typical acquisition time of $300 \mathrm{~s}$. Bias voltages $\mathrm{V}$ refer to the sample voltage with respect to the tip. ${ }^{[14-16]}$ 


\section{Results and Discussion}

\subsection{Chiral Self-assembly of Organic Molecules on Au(111)}

Surface nanostructuring by molecular self-organization is a relevant process in the growing field of nanotechnology. Depending on the characteristics of the molecules and on the type of interactions among them and with the substrate, a variety of surface patterns have been observed by means of scanning tunneling microscopy (STM). ${ }^{[17]}$ An early contribution to this field were our investigations on the selfassembly of the aromatic molecule 1-nitronaphthalene (NN, Fig. 1, inset) adsorbed on the reconstructed $\mathrm{Au}(111)$ surface. ${ }^{[18]}$ The confinement of $\mathrm{NN}$ to two dimensions introduces chirality not present in the gas phase. On the surface both enantiomers are present in equal amounts, thus forming a racemic mixture. The $\mathrm{Au}(111)$ sample was held at room temperature during the deposition and below $50 \mathrm{~K}$ during the measurements. In order to minimize tip-induced motion of the molecules at submonolayer coverages $^{[6,19,20]}$ small tunneling currents ( $\mathrm{I}=10 \mathrm{pA})$ were used.

At coverages between 0.05 and 0.15 monolayers (ML) self-assembled quasiOD clusters of distinct size and structure appear at the fcc-elbows of the reconstruction (Fig. 1b). At slightly higher coverage (0.2 ML) identical clusters are observed within fcc-domains (Fig. 1c) and, sporadically, within hcp domains and on domain walls. All decamers appear identical in the STM images, except for a mirror symmetry (clusters 1 and 2 in Fig. 1c). They consist of an 8-molecule ring surrounding a 2-molecule core. Manipulation experiments at decreased tunneling resistance show that the decamers behave like stable 'supermolecules' ${ }^{[12]}$ We conclude that the structure and stability of the decamers is determined by highly specific intermolecular forces, while the interaction of these 'supermolecules' with the reconstructed substrate and a mutual repulsion at small distances determine their lateral spacing.

At medium coverage (0.3-0.75 ML) the growth mode changes to the formation of 1D molecular double chains (see Fig. 8a at 0.7 ML, Section 3.2) guided by the reconstruction domains or by step edges. At full monolayer coverage 1D and 2D periodic molecular structures coexist on the surface. ${ }^{[5]}$ At a tunneling voltage $U=-2.3$ $\mathrm{V}$, intramolecular structure is resolved and the contrast pattern shows the expected handedness. ${ }^{[2]}$ The charge density calculated for the highest occupied molecular orbital (HOMO) of the free NN molecule closely resembles the observed sub-molecular structure. ${ }^{[2]}$ At lower bias voltage hybridization with Au-substrate states results in a nearly symmetric appearance of the molecules ${ }^{[21]}$ where the long axis of the ellipsoids coincides with the long axis of the naphthalene core.

Molecular dynamics simulations rationalize the observed supramolecular arrangements. ${ }^{[2]}$ Within each single strand the molecules are arranged in a 'head-totail' configuration via hydrogen bonds between a negatively charged oxygen atom and the 'backside' hydrogen atom at a carbon atom of a neighbouring NN molecule. The second strand is rotated by $180^{\circ}$ and shifted by half a period such that opposite charges are close to each other. The double chains are positively charged outside and therefore mutually repel as observed experimentally. We conclude that straight, defect-free segments of the double chains consist of exclusively one NN enantiomer thus representing a $1 \mathrm{D}$ conglomerate. ${ }^{[2]}$ Below a critical density of molecules quasi-0D decamers are energetically more favorable than linear double chains with unsaturated hydrogen bonds at their ends. In the decamers a homochiral molecular ring along the periphery is stabilized by a core composed of two molecules with opposite chirality.

Our STM observations and theoretical

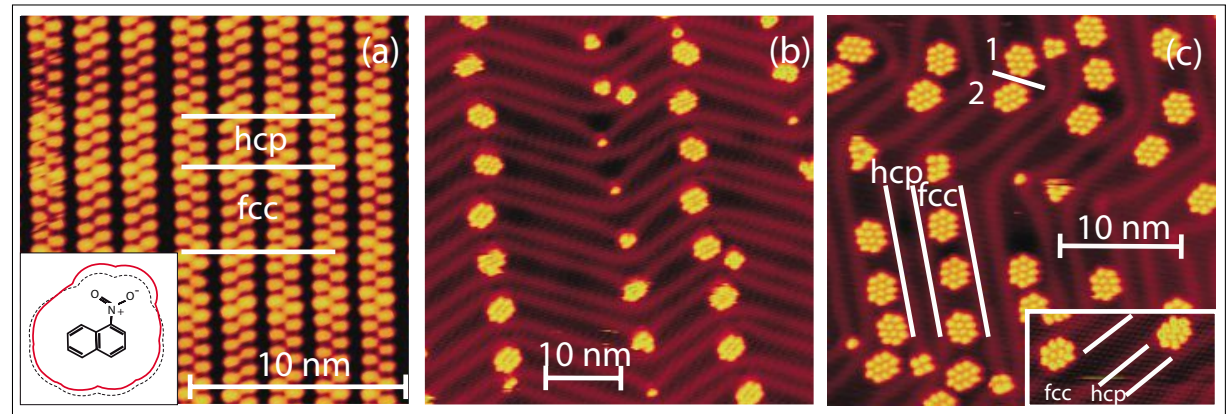

Fig. 1. STM images at $50 \mathrm{~K}$ of a reconstructed $\mathrm{Au}(111)$ surface with adsorbed NN; (a) $0.7 \mathrm{ML}$ NN. Inset: Structural formula of NN. The dashed line encloses the 'exclusion' area resulting from steric repulsion. The distance of the full line from the dashed line indicates the strength of a negative electrostatic potential computed on the dashed line. (b) $0.1 \mathrm{ML} N \mathrm{~N}$ at $65 \mathrm{~K}$. (c) $0.2 \mathrm{ML}$ NN at 50 K. Inset: 0.2 ML NN at $10 \mathrm{~K}$. Reprinted with permission from M. Böhringer, K. Morgenstern, W.-D. Schneider, R. Berndt, F. Mauri, A. De Vita, R. Car, Phys. Rev. Lett. 1999, 83, 324; http://link.aps. org/abstract/PRL/v83/p324. Copyright American Physics Society 1999. modeling led to a detailed understanding of the stability and the internal geometry of self-assembled supramolecular structures of the 2D chiral molecule 1-nitronaphthalene on $\mathrm{Au}(111)$. Recently, these tools allowed us to understand the supramolecular self-assembly and selective step decoration of these molecules on the $\mathrm{Au}(111)$ surface $^{[22]}$ and to follow in real space a coverage-driven chiral phase transition from a conglomerate to a racemate. ${ }^{[4,23]}$ Moreover, the formation of surface-supported supramolecular structures whose size and aggregation pattern were rationally controlled by tuning the non-covalent interactions between individual absorbed molecules has been reported recently for substituted porphyrin molecules adsorbed on a gold surface. [24]

As a second example for 2D-supramolecular self-assembly the aromatic hydrocarbon rubrene $(5,6,11,12$-tetra phenylnaphthacene, $\mathrm{C}_{48} \mathrm{H}_{28}$ ) is chosen. This molecule is chiral and non-planar due to intramolecular steric hindrance, $[25,26]$ which causes a twisting of the central tetracene-backbone around its axis and forces the four phenyl-groups to rotate out of the backbone plane. Its highly efficient luminescence established the success of rubrene as a dopant for organic light-emitting diodes to improve the efficiency and stability of the devices. ${ }^{[27]}$ Despite this growing interest in the application of rubrene, there have been no nanoscale investigations of the self-assembly process and the molecular and supramolecular chirality of rubrene until recently. ${ }^{[8]}$ Fig. 2 shows the surprising hierarchical complexity of the nested twodimensional supramolecular assembly of rubrene on gold with its three successive generations: single molecules, pentagonal supermolecules and supramolecular decagons. Recently, theoretical modeling has shown that the self-assembly of rubrene pentagons into 1D chains and rings is due to the competition between short-range attractive van der Waals forces and longrange repulsive interactions. The latter arise caused by charge transfer from the metallic substrate to the molecules leading to the formation of mutually repulsive standing dipoles. ${ }^{[13]}$ In the hierarchical assembly steps the chirality of the individual molecules is conserved. Due to the chirality-dependent rotation of the building blocks, both resulting generations of the progression are chiral on their own. Since this self-organization of increasing complexity is enantioselective on the molecular and supramolecular level, we obtain a spontaneous resolution of the original racemic mixture of individual molecules into homochiral architectures. Our findings on the conservation and recognition of chirality on all stages of the supramolecular selfassembly of rubrene may have an impact 


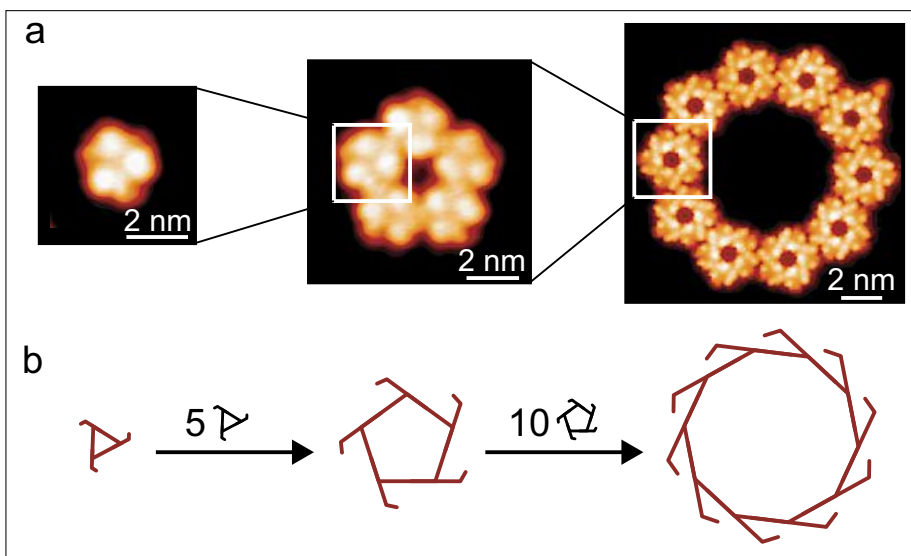

on the development of chiral molecular electronic and optoelectronic devices and exemplify the working principle of basic processes in nature.

A very interesting aspect in this context is the three-dimensional chirality transfer in rubrene multilayer islands. ${ }^{[11]}$ Multilayer islands up to a thickness of six layers on a $\mathrm{Au}(111)$ surface have been investigated. The molecules self-organize in parallel twin rows, forming mirror domains of defined local structural chirality. Each layer is composed of twin-row domains of the same structural handedness rotated by $120^{\circ}$ with respect to each other. Moreover, this structural chirality is transferred to all successive layers in the island, resulting in the formation of three-dimensional objects having a defined structural chirality. The centered rectangular surface unit cell differs from the one characteristic for the single-crystal orthorhombic phase.

Two-dimensional (2D) tiling constitutes a fundamental issue in topology, [28] with fascinating examples in nature and art, and applications in many domains such as cellular biology, ${ }^{[29]}$ foam physics, ${ }^{[30]}$ or crystal growth. ${ }^{[31]}$ Recently, we presented the first observation of surface tiling with both non-periodic and periodic arrangements of slightly distorted pentagons, hexagons, and heptagons formed by rubrene molecules adsorbed on a $\mathrm{Au}(111)$ surface.[10] On adjacent regions of the sample, ordered honeycomb and hexagonal close-packed patterns are found. The existence of manifold arrangements in the supramolecular self-assembly of rubrene on gold originates from the three-dimensional non-planar flexible structure of the molecule, as well as from the nature of the intermolecular bonds.

A detail of the latter non-periodic phase is shown in Fig. 3a. The dashed blue circle surrounds a single rubrene molecule, with the sub-molecular contrast revealing three lobes and a quite regular three-fold symmetry. ${ }^{[8,9]}$ The self-assembled pattern is composed of supramolecular pentagons, hexagons and filled heptagons, which ap-
Fig. 2. Hierarchy and conservation of chirality in the spontaneous two-dimensional supramolecular assembly. a) STM: A detail of the latter non-periodic phase shows images representing the three generations of the nested two-staged self-organization. From left to right: single molecule, pentagonal supermolecule, supramolecular decagon. Typical tunneling parameters are $\mathrm{I}=20 \mathrm{pA}$ and $\mathrm{U}=$ -0.8 V. b) From left to right: Enantioselective assembly from L-type monomers to L-type pentagons and further on to L-type de-cagons. Reprinted with permission from M.-C. Blüm, E. Ćavar, M. Pivetta, F. Patthey, W.-D. Schneider, Angew. Chem. Int Ed. 2005, 44, 5334.

Copyright John Wiley \& Sons 2005.

pear to be randomly distributed over the surface. There exist 11 distinct tilings by regular polygons, ${ }^{[28]}$ however, a combination of regular pentagons, hexagons, and heptagons generates empty gaps and overlapping regions, as inferred from the consideration of the angles at the corners of a regular pentagon $\left(108^{\circ}\right)$, hexagon $\left(120^{\circ}\right)$ and heptagon $\left(\approx 129^{\circ}\right)$. Only in the case of joining three hexagons with one common corner, is the angular sum exactly $360^{\circ}$. Nevertheless, there exist three configurations yielding an angular sum close to $360^{\circ}$, schematized in Fig. 3 b: pentagon-hexagon-heptagon $\left(\approx 357^{\circ}\right)$, pentagon-heptagon-heptagon $\quad\left(\approx 366^{\circ}\right)$, and hexagon-hexagon-heptagon $\left(\approx 369^{\circ}\right)$. The introduction of a slight distortion of the polygons allows a plane-filling tessellation. These three configurations are the most frequently realized in the non-periodic supramolecular tiling shown in Fig. 3a. An example of each combination is superimposed on the STM image. As expected, the fact that the angular sum of the polygons involved in the patterning is either smaller or larger than the required $360^{\circ}$ for a true plane-filling leads to small distortions of the side lengths and the angles of the objects, compared to regular polygons. The STM image in Fig. 3a also reveals that the supramolecular heptagons accommodate an additional molecule in their center. These captured specimens present two different appearances, highlighted in Fig.

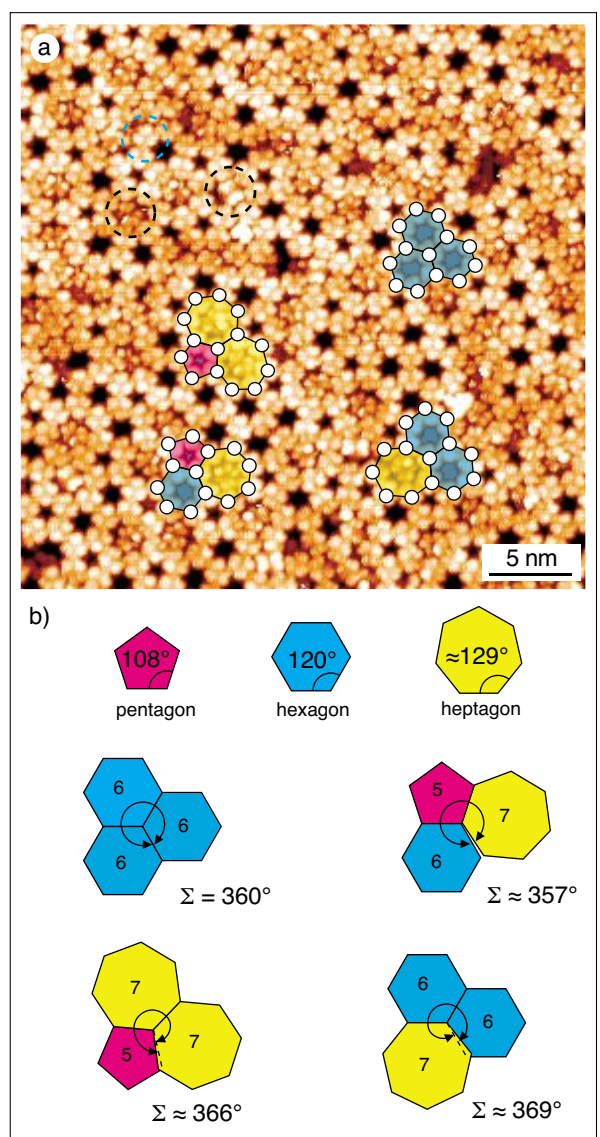

Fig. 3. a) STM image showing a zoom of tiling of type $B$ formed by a non-periodic assembly of pentagons, hexagons, and filled heptagons. b) Schematic representation of the combinations of pentagons, hexagons, and heptagons resulting in three configurations close to the case of an ideal honeycomb arrangement. Reprinted with permission from M. Pivetta, M.-C. Blüm, F. Patthey, W.-D. Schneider, Angew. Chem. Int. Ed. 2008, 47, 1076. Copyright John Wiley \& Sons 2008.

$3 \mathrm{a}$ by the two dashed black circles. The two conformations are presumably due to the varying small deformations of the heptagons, offering more circular or more elliptical holes for the additional inserted molecules. An arrangement of pentagons, hexagons and heptagons which is periodic throughout a whole domain is realized in another type of supramolecular phase present in this mixed molecular layer (not shown). ${ }^{[10]}$

A beautiful singularity existing inside these perfectly periodic domains is shown in the STM image of Fig. 4a. The honeycomb pattern is locally interrupted by a circular replacement of twelve hexagons by a ring of six pentagon-heptagon pairs, surrounding a core of seven hexagons. These rosettes are preferentially formed at elbow sites of the herringbone reconstruction of the substrate. In this image three such rosettes are present, with dashed blue circles highlighting two of them. A schematic representation of the rosette structure superimposed to the third pattern reveals that this symmetric structure with six 


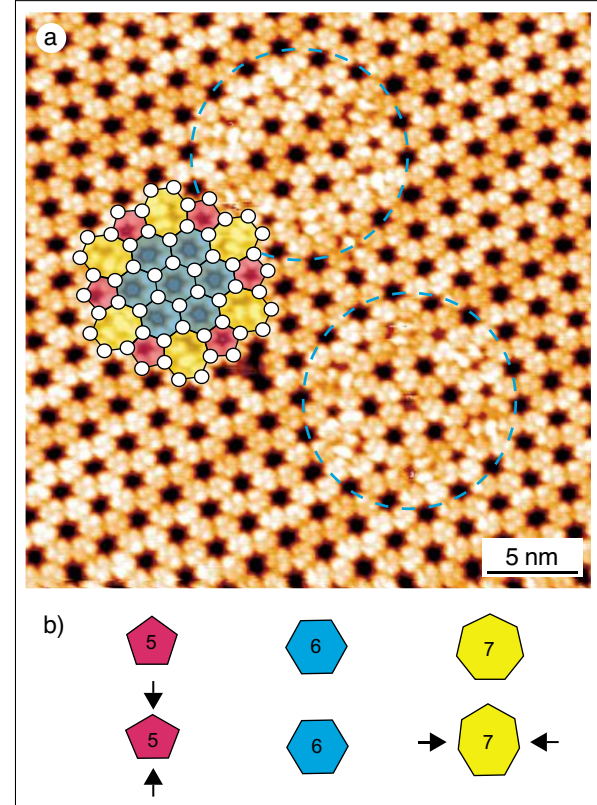

Fig. 4. a) STM image showing three supramolecular rosettes embedded in a well-ordered honeycomb domain. The schematic drawing of the pattern is superimposed on one of the three rosettes. b) Schematic representation of the distortion of the pentagons and heptagons to compose the tiling. Reprinted with permission from M. Pivetta, M.-C. Blüm, F. Patthey, W.-D. Schneider, Angew. Chem. Int. Ed. 2008, 47, 1076. Copyright John Wiley \& Sons 2008.

outer pentagons and six heptagons forms a regular hexagon, which is perfectly inserted into the surrounding honeycomb arrangement. However, the ring of pentagons and heptagons suffer from the constriction from two sides due to the presence of an inner ring as well as an outer ring of hexagons. Moreover, on the outer side of the rosette the combination pentagon-hexagonhexagon is present which - with a nominal angular sum of $348^{\circ}$ - induces a more important deformation of the pentagons. This angular stress leads to a compression of both the pentagons and the heptagons in opposite directions, as illustrated in Fig. 4b. Again, within the supramolecular rosettes the heptagons are filled with additional molecules, exhibiting mainly the elongated conformation. We note that the replacement of two hexagons with a pentagon-heptagon pair is one of the most common topological defects found in honeycomb structures, such as in graphite and in carbon nanotubes. ${ }^{[32]}$ The replacement of all hexagons would generate the so-called pentaheptite structure, a tiling observed in crystallography. [33]

In the sub-monolayer regime, the selfassembly of rubrene on $\mathrm{Au}(111)$ results in a variety of distinct phases. As a function of the molecular coverage, honeycomb islets, supramolecular pentagonal chains, and close-packed islands have been observed. ${ }^{[8,9]}$ The periodic and non-periodic tilings presented here demonstrate in an especially fancy manner the conformational flexibility of the rubrene molecules. Supramolecular self-assembly results from the contributions of molecule-substrate and molecule-molecule interactions. Intermolecular bonds are mainly based on weak, non-covalent interactions such as dipole-dipole (or higher order multipole) forces, hydrogen bonds, or van der Waals (vdW) interactions. ${ }^{[17,34]}$ Given the absence of a dipole moment, the bonding between rubrene molecules might have contributions from electrostatic quadrupole-quadrupole interactions, vdW forces, $\pi-\pi$ and $\mathrm{CH}-\pi$ bonding. Among them vdW forces are isotropic, the other interactions are directional. While the $\pi$ - $\pi$ interaction favours a parallel arrangement of the $\pi$-systems, ${ }^{[35]}$ the $\mathrm{CH}-\pi$ bond is particularly strong for a $\mathrm{CH}$ group pointing perpendicular to a $\pi$ system of another molecule. ${ }^{[35]}$ These forces relying on the existence of $\pi$ systems are effectively realized between rubrene molecules, due to the non-planar aromatic tetracene backbone, the four phenyl groups, and the presence of $28 \mathrm{CH}$ bonds pointing into three dimensions. The directional interactions can account for the formation of the ordered hexagonal structures, in which the bond angle between molecules is precisely $120^{\circ}$. Intriguingly, a small modification of the intermolecular bonds or a slight change in the molecular conformation results in the formation of pentagons and heptagons. The adaptive behaviour of rubrene allows these modifications which leads to the observed 2D periodic and non-periodic tilings with pentagons, hexagons, and heptagons.

The fact that the supramolecular selfassembly of rubrene on $\mathrm{Au}(111)$ results in different adsorption conformations of the physisorbed molecules is reflected in a different electronic structure of the adsorbates. Owing to the three-dimensional geometry of the molecule providing an inherent decoupling of the molecular $\pi$ states from the substrate the conformers are distinguished by their submolecular appearance in the scanning tunneling microscopy images and by the corresponding position of the highest molecular orbital in the differential conductance $(\mathrm{dI} / \mathrm{dV})$ spectra. The application of an electric field induces a switching of the electronic and geometric conformation of the self-assembled molecules. ${ }^{[9]}$

\subsection{STM-induced Light Emission from Fullerene Molecules on a Dielectric Substrate}

Light emission induced by electrons tunneling through the junction formed by the sample and the tip of a STM has been proposed to characterize the optical properties of nanoscale objects at surfaces. ${ }^{[36,37]}$ Contrary to conventional non-local tech-

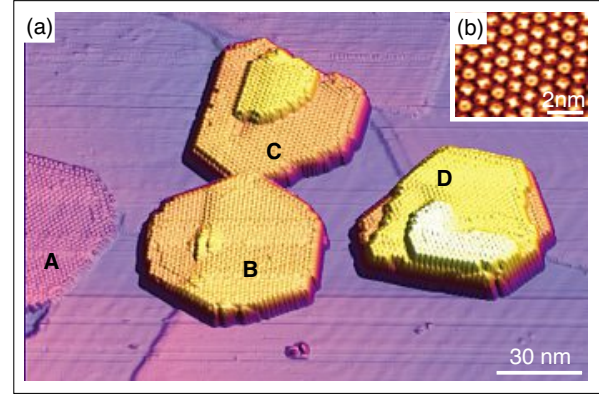

Fig. 5. (a) STM image of $C_{60}$ nanocrystals formed on a $\mathrm{NaCl}$ ultrathin film grown on $\mathrm{Au}(111)(\mathrm{U}=-3 \mathrm{~V}, \mathrm{I}=0.02 \mathrm{nA})$. Island $\mathrm{A}$ is a $\mathrm{C}_{60}$ monolayer on $\mathrm{Au}(111)$, the small blue triangle below $A$ is part of the bare Au surface. Hexagonal island $B$, and truncated triangular islands $C$ and $D$ consist of up to two, three, and four $\mathrm{C}_{60}$ molecular layers, respectively, on $\mathrm{NaCl}$. (b) Sub-molecular resolution on island $\mathrm{B}$ $(U=-3 \mathrm{~V}, \mathrm{I}=0.1 \mathrm{nA})$. Reprinted with permission from E. Ćavar, M.-C. Blüm, M. Pivetta, F. Patthey, M. Chergui, W.-D. Schneider, Phys. Rev. Lett. 2005, 95, 196102; http://link.aps. org/abstract/PRL/v95/e196102. Copyright American Physics Society 2005.

niques, the local character of this method offers the unique possibility to select and probe individual atoms, molecules or clusters on surfaces. Luminescence from

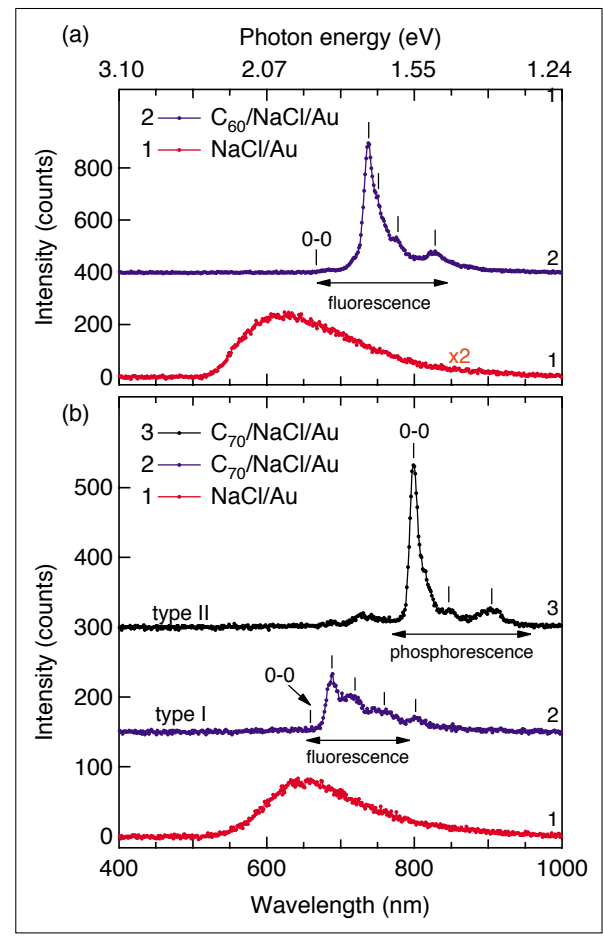

Fig. 6. (a) STM-LE spectra acquired over a $C_{60}$ nanocrystal (spectrum 2, $\mathrm{U}=-3 \mathrm{~V}, \mathrm{I}=4 \mathrm{nA}, \mathrm{t}=$ $60 \mathrm{~s}$ ) and over the underlying $\mathrm{NaCl}$ film (spectrum $1, \mathrm{U}=-3 \mathrm{~V}, \mathrm{I}=2 \mathrm{nA}, \mathrm{t}=60 \mathrm{~s}$ ). (b) STM-LE spectra acquired over a $C_{70}$ nanocrystal (spectra 2 and 3) and over the $\mathrm{NaCl}$ film (spectrum 1), $(U=-3 V, I=1 \mathrm{nA}, t=60 \mathrm{~s})$. Spectra are vertically shifted for clarity. $0-0$ indicates the pure electronic origins. Reprinted with permission from F. Rossel, M. Pivetta, F. Patthey, W.D. Schneider, Optics Express 2009, 17, 2714. Copyright The Optics Society 2009. 


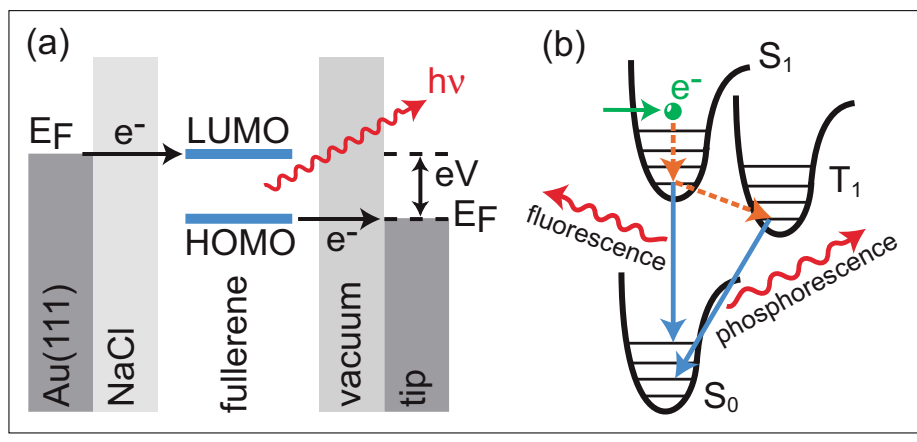

Fig. 7. (a) Schematic energy diagram of a double-barrier tunnel junction at negative bias voltage, corresponding to the conditions for luminescence. The simultaneous population and depopulation of the LUMO and HOMO by tunneling electrons creates an electronically and vibrationally excited state of the molecule. (b) Fluorescence channel: the molecule relaxes to the ground vibrational state of the lowest electronic excited singlet state $\mathrm{S} 1$ followed by a radiative transition to the singlet ground state S0. Phosphorescence channel: a radiationless transition occurs between the singlet state $\mathrm{S} 1$ and triplet state $\mathrm{T} 1$ with subsequent radiative molecular transition to the ground state S0. Reprinted with permission from F. Rossel, M. Pivetta, F. Patthey, W.-D. Schneider, Optics Express 2009, 17, 2714. Copyright The Optics Society 2009.

supported molecules has been obtained by successfully decoupling them from the metallic substrate in order to avoid quenching of the radiative transitions, ${ }^{[38-41]}$ using either a thin oxide film ${ }^{[38]}$ or several molecular layers. ${ }^{[39]}$ Recently, Ćavar et al. and Rossel et al. presented the first observation of energy-resolved luminescence from supported $\mathrm{C}_{60}$ and $\mathrm{C}_{70}$ molecules, respectively. ${ }^{[14,15]}$

Fig. 5 shows $\mathrm{C}_{60}$ islands grown on both the bare $\mathrm{Au}(111)$ (A) and the $\mathrm{NaCl}$ covered surface (B-D). The nanocrystals present a minimum height of two layers of $\mathrm{C}_{60}$ molecules (island $\mathrm{B}$ ). The nucleation of the $\mathrm{C}_{60}$ nanocrystals starts at defects of the $\mathrm{NaCl}$ layer (protrusions or vacancies), monatomic steps of $\mathrm{Au}(111)$ (covered with $\mathrm{NaCl}$ ), or edges of the second layer of $\mathrm{NaCl}$. The $\mathrm{C}_{60}$ molecules form hexagonally arranged layers with an intermolecular distance of $1 \mathrm{~nm}$.

Fig. 6 shows STM-induced light emission (STM-LE) spectra acquired over a $\mathrm{C}_{60}$ (a) and a $\mathrm{C}_{70}$ (b) nanocrystal, as well as over the $\mathrm{NaCl}$ spacer layers. Luminescence from the supported fullerenes, clearly distinguishable from the localized surface plasmon (LSP) emission, is observed for negative excitation voltages larger than $-2.3 \mathrm{~V}$ for $\mathrm{C}_{60}$ and $-2.5 \mathrm{~V}$ for $\mathrm{C}_{70}$. For positive voltages up to $+5 \mathrm{~V}$, no photon emission was detected for both fullerenes.

The observed bias dependence of the STM-induced light emission from the fullerene molecules is characteristic of a hot electron injection mechanism ${ }^{\text {[38,39] }}$ followed by a radiative decay associated with the highest occupied molecular orbital-lowest unoccupied molecular orbital (HOMO-LUMO) gap of the molecules. This interpretation implies that the energy levels of the emitting molecules are not pinned to the gold substrate, but shift with the applied bias, as illustrated in Fig. 7a. When the substrate is negatively biased with a voltage above the observed threshold value, the LUMO falls at an energy level lower than the Fermi energy $\left(\mathrm{E}_{\mathrm{F}}\right)$ of the sample and the HOMO at an energy level higher than the Fermi energy of the tip. Electrons tunnel elastically from the gold substrate into the LUMO through the $\mathrm{NaCl}$ barrier (hot electron injection) and simultaneously from the HOMO into the tip through the vacuum barrier to create electronically and vibrationally excited states of molecules. The transitions to the ground state give rise to the observed molecular luminescence. The fact that the latter is not observed for positive excitation voltages may be due to an asymmetry of the HOMO and LUMO position with respect to $\mathrm{E}_{\mathrm{F}}$ and to different characteristics for the tip-molecule and molecule-substrate double barrier. ${ }^{[14,42]}$ In order to identify the electronic transitions giving rise to the observed molecular light emission spectra shown in Fig. 6, we compare our results with laser-induced photoluminescence data from fullerene molecules in different media.

Photoluminescence spectra acquired for $\mathrm{C}_{60}$ in rare gas matrices, ${ }^{[43,44]}$ thin films, ${ }^{60}{ }^{60,46]}$ and single crystals ${ }^{[47,48]}$ have been considered. This comparison permits the observed spectra to be attributed to fluorescence from the $\mathrm{S} 1 \rightarrow \mathrm{S} 0$ transition. In the light of the present results on the plasmon-mediated amplification, the previously reported STM-induced luminescence from $\mathrm{C}_{60}$ molecules ${ }^{[14]}$ has to be reinterpreted. We interpret the observed luminescence spectra as fluorescence from $\mathrm{C}_{60}$, although a contribution from a phosphorescence channel, corresponding to the
$\mathrm{T} 1 \rightarrow \mathrm{S} 0$ transition, cannot be excluded in the low energy part of the spectra.

Intriguingly, the observation of both fluorescence and phosphorescence is realized in the case of $\mathrm{C}_{70}$, as deduced from the comparison of our results with laserinduced photoluminescence data from dispersed $\mathrm{C}_{70}$ molecules in different media ${ }^{[49]}$ and from $\mathrm{C}_{70}$ solids. ${ }^{50-52]}$ The observed spectra of type I are attributed to fluorescence from the $\mathrm{S} 1 \rightarrow \mathrm{S} 0$ transition and the spectra of type II to phosphorescence from the $\mathrm{T} 1 \rightarrow \mathrm{S} 0$ transition. Because of a relatively low spectral resolution in the present experiment $(8 \mathrm{~nm})$, only the pure electronic origin (0-0) corresponding to the triplet-to-singlet ground state transition is identified at about $800 \mathrm{~nm}$. The other spectral features correspond to unresolved multiplet vibronic structures. Note that the broad peak at $800 \mathrm{~nm}$ in the spectrum of type I shown in Fig. 6(b) is assigned to the phosphorescence channel rather than to the fluorescence one. We indeed observed several times emission spectra consisting of a superposition of components from both radiative transitions.

The plasmon-enhancement phenomenon, also at the heart of tip-enhanced Raman spectroscopy (TERS), ${ }^{[53]}$ contributes essentially to the high spatial resolution of the STM-LE technique, since only luminescence from molecules lying within the spatial extension of the localized surface plasmon is subject to be enhanced. The observed site-dependent rigid shift of the entire luminescence spectra of $\mathrm{C}_{60}$ and $\mathrm{C}_{70}$ nanocrystals ${ }^{[15]}$ gives direct experimental evidence of the local character of the technique in the presence of an ensemble of molecules, since it is likely to be related, respectively, to the intermolecular orientational correlation in a pair of $\mathrm{C}_{60}$ molecules over which the excitation is delocalized ${ }^{[54-56]}$ and to the sensitivity of the lowest excited electronic states of the C70 molecule to the environment in which it is trapped. ${ }^{[57,58]}$ The weak interactions with the surrounding molecules may vary with the probed sites due to orientational disorder and to the presence of lattice defects.

It is interesting to note that one $\mathrm{ML}$ thick fullerene nanocrystals on $\mathrm{NaCl}$ have been observed, although very rarely. STMLE spectra acquired on these extremely thin crystals clearly show the signature of the molecular luminescence. A different behavior has been reported for pentacene nanocrystals grown on $\mathrm{KCl}$ on $\mathrm{Au}(111)$ : no luminescence has been found for crystals thinner than $1.5 \mathrm{~nm}$ (3 ML). ${ }^{[59]}$ Only bulk-like excitonic features have been observed in the optical spectra demonstrating the dominance of inter-molecular coupling even at the nanocrystal limit for pentacene.

The luminescence of nanocrystals composed of a mixture of both fullerene species 
has also been investigated. The sample was prepared by simultaneous sublimation of high purity powders of each type of fullerene and the selected molecules in the surface layer were identified as $\mathrm{C}_{60}$ and $\mathrm{C}_{70}$ by comparing the STM-imaged orbitals with DFT calculations.[60] The interesting observation is that the obtained spectra from the two mixed species are very similar and strongly resemble the fluorescence spectrum of a $\mathrm{C}_{70}$ nanocrystal. Although a modification of the molecular electronic levels towards the electronic structure of a mixed fullerene crystal cannot be excluded, the present finding indicates that the molecular luminescence induced by tunneling electrons does not originate from an individually selected molecule in the top layer, in contrast to what was proposed in ref. [14]. The excitation and emission processes occurring in such a molecular nanocrystal can be accompanied by non-radiative energy transfer between molecules.[61-63] Consequently, the light emission location will be partly spatially separated from the excitation location. Luminescence will only be observed if its origin lies within the spatial extension of the localized surface plasmon.

The fact that STM-induced optical spectra do not necessarily originate from the sole selected molecule suggests some limits of the STM-LE technique. The situation could be different for molecular impurities, embedded in a guest molecular crystal with completely different electronic structure: in this case the host molecule possibly behaves as an isolated molecule. Thus, for molecules in an ensemble, such as for example nanocrystals composed of a mixture of $\mathrm{C}_{70}$ and $\mathrm{C}_{60}$ fullerenes, an unambiguous chemical recognition on the single-molecular scale is not possible. The spatial resolution limit is related to the spatial extension of the tip-induced LSP, which in turn depends on the tip geometry. Consequently, considering a typical etched tip, the spatial resolution of the technique on molecular layers is expected to be $\approx 5 \mathrm{~nm}$.

This value is confirmed in the following experiment, carried out on fullerene nanocrystals prepared by successive sublimation of $\mathrm{C}_{70}$ and $\mathrm{C}_{60}$ molecules. With this procedure, nanocrystals formed by a central part of $\mathrm{C}_{70}$ and a rim of $\mathrm{C}_{60}$ can be obtained, see Fig. 8. ${ }^{[60]}$ In the STM-LE measurements, the sole molecular signature of the considered area is identified down to a distance of about 1.5-2 $\mathrm{nm}$ from the boundary between both molecular species, as shown in Fig. 8. Phosphorescence associated with the transition $\mathrm{T} 1 \rightarrow \mathrm{S} 0$ within a $\mathrm{C}_{70}$ molecule (Fig. 8c, spectrum 1) and fluorescence associated mainly with the transition $\mathrm{S}^{\prime} \rightarrow \mathrm{S} 0$ within $\mathrm{a} \mathrm{C}_{60}$ dimer (Fig. $8 \mathrm{c}$, spectrum 2) are clearly identified.

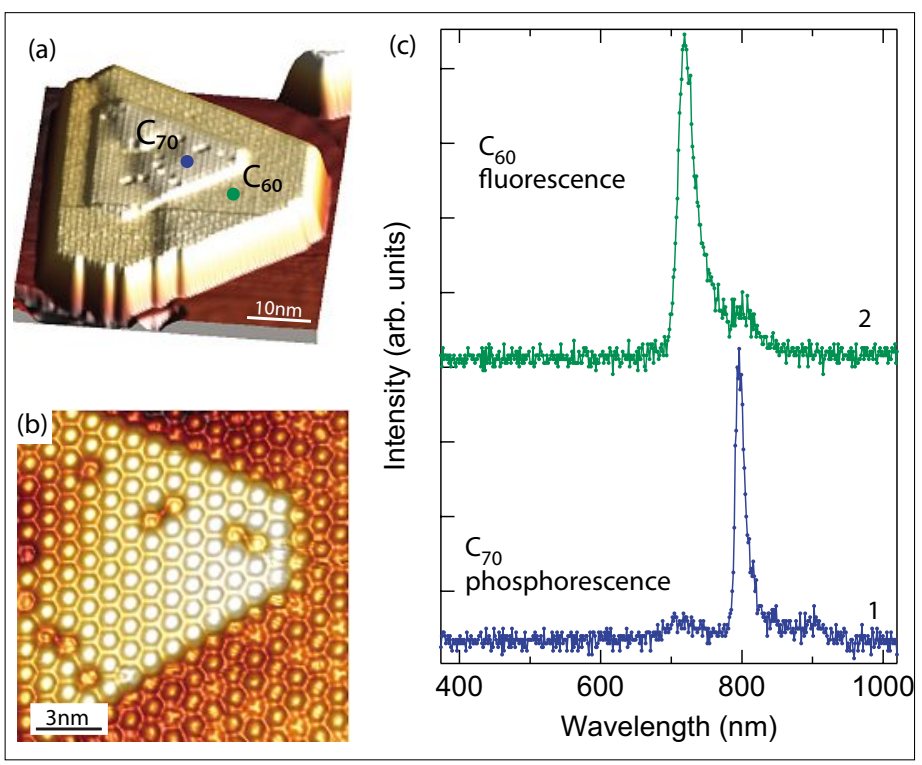

Fig. 8. (a) STM image of a fullerene nanocrystal composed of both fullerene species prepared by successive evaporations (3D image processing150), ( $\mathrm{U}=-3 \mathrm{~V}, \mathrm{I}=0.02 \mathrm{nA})$. (b) Submolecularly resolved topography of the surface layer of the nanocrystal in (a) (3D image processing $\left.{ }^{[62]}\right)$, $(U=+3 \mathrm{~V}, \mathrm{I}=0.02 \mathrm{nA})$. (c) STM-LE spectra acquired while selecting one $\mathrm{C}_{70}$ (spectrum 1) and one $\mathrm{C}_{60}$ (spectrum 2) molecule in the surface layer displayed in (b), $(\mathrm{U}=-3 \mathrm{~V}, \mathrm{I}=1 \mathrm{nA}, \mathrm{t}=60 \mathrm{~s})$. Reprinted with permission from F. Rossel, M. Pivetta, W.-D. Schneider, Surf. Sci. Rep. 2010, 65,129. Copyright Elsevier 2009.

In summary, light emission induced by tunneling electrons was observed from $\mathrm{C}_{60}$ and $\mathrm{C}_{70}$ molecules that were electronically decoupled from the metal substrate by a thin $\mathrm{NaCl}$ film. The molecular transitions giving rise to light emission were identified by comparison with laser-induced photoluminescence data from dispersed molecules or molecular crystals reported in the literature. The key role of localized surface plasmons in the enhancement of molecular emission is unambiguously demonstrated. The present observation of local fluorescence and phosphorescence represents a crucial step towards chemical recognition on the nanoscale. ${ }^{[16]}$

\section{Conclusion}

In this short review experiments at the scale of molecules, and supermolecules have been presented, which exhibit exciting physical effects. These include light emission from supported molecules, twodimensional self-assembly and the formation of chiral supramolecular architectures. The common link between these different topics is the characterization of the structural and electronic properties of supported nanostructures at atomic-scale spatial and sub-meV-scale spectral resolution. The opportunities of this concept in the emerging field of nanotechnology, where size matters, are evident. For example, a reduction in size often provides an increase in speed for electronic or magnetic devices.
Therefore, a further advancement towards future nanotechnology will depend crucially on the precise control, characterization, and, ultimately, functionalization, of matter at the atomic and molecular level. This prospect remains an inciting scientific and technological challenge.

\section{Acknowledgments}

I would like to thank F. Patthey, M. Pivetta, R. Berndt, R. Gaisch, M. Böhringer, K. Morgenstern, M.-C. Blüm, E. Ćavar, C. Rossel, M. Chergui, K. Glöckler, M. Sokolowski, E. Umbach, J. K. Gimzewski, B. Reihl, R. R. Schlittler, M. Tschudy, F. Mauri, A. De Vita, M. Stengel, R. Car, G. Tomba, M. Vladimirova, A. P. Seitsonen, and A. Baldereschi for a very fruitful and stimulating collaboration. This work has been supported by the Swiss National Science Foundation.

Received: July 26, 2011

[1] G. M. Whitesides, B. Grzybowski, Science 2002, 295, 2418 .

[2] M. Böhringer, K. Morgenstern, W.-D Schneider, R. Berndt, F. Mauri, A. De Vita, R. Car, Phys. Rev. Lett. 1999, 83, 324.

[3] M. Böhringer, K. Morgenstern, W.-D Schneider, R. Berndt, Angew. Chem. 1999, 111 , 832; Angew. Chem. Int. Ed. 1999, 38, 821.

[4] M. Böhringer, W.-D. Schneider, R. Berndt, Angew. Chem. 2000, 112, 821; Angew. Chem. Int. Ed. 2000, 39, 792.

[5] M. Böhringer, K. Morgenstern, W.-D Schneider, M. Wühn, C. Wöll, R. Berndt, Surf. Sci. 2000, 444, 199.

[6] M. Böhringer, K. Morgenstern, W.-D Schneider, R. Berndt, Surf. Sci. 2000, 457, 37.

[7] M. Böhringer, R. Berndt, W.-D. Schneider, Chem. in unserer Zeit 2005, 39, 326. 
[8] M.-C. Blüm, E. Ćavar, M. Pivetta, F. Patthey, W.-D. Schneider, Angew. Chem. Int. Ed. 2005 , 44, 5334.

[9] M.-C. Blüm, M. Pivetta, F. Patthey, W.-D. Schneider, Phys. Rev. B 2006, 73, 195409.

[10] M. Pivetta, M.-C. Blüm, F. Patthey, W.-D. Schneider, Angew. Chem. Int. Ed. 2008, 47, 1076.

[11] M. Pivetta, M.-C. Blüm, F. Patthey, W.-D. Schneider, J. Phys. Chem B 2009, 113, 4578.

[12] M. Pivetta, M.-C. Blüm, F. Patthey, W.-D. Schneider, ChemPhysChem 2010, 11, 1558.

[13] G. Tomba, M. Stengel, W.-D. Schneider, A. Baldereschi, A. De Vita, ACSNano 2010, 12, 7545.

[14] E. Ćavar, M.-C. Blüm, M. Pivetta, F. Patthey, M. Chergui, W.-D. Schneider, Phys. Rev. Lett. 2005, 95, 196102.

[15] F. Rossel, M. Pivetta, F. Patthey, W.-D. Schneider, Optics Express 2009, 17, 2714.

[16] F. Rossel, M. Pivetta, W.-D. Schneider, Surf. Sci. Rep. 2010, 65, 129.

[17] a) S. De Feyter, F. C. De Schryver, Chem. Soc. Rev. 2003, 32, 139; b) J. V. Barth, Annu. Rev. Phys. Chem. 2007, 58, 375.

[18] J. V. Barth, H. Brune, G. Ertl, R. J. Behm, Phys. Rev. B 1990, 42, 9307.

[19] M. Böhringer, W.-D. Schneider, R. Berndt, Surf. Sci. 1998, 57, 72.

[20] M. Böhringer, W.-D. Schneider, R. Berndt, K. Glöckler, M. Sokolowski, E. Umbach, Phys. Rev. B 1998, 57, 4081.

[21] A. J. Fisher, P. E. Blöchl, Phys. Rev. Lett. 1993, $70,3263$.

[22] M. Vladimirova, M. Stengel, A. De Vita, A. Baldereschi, M. Böhringer, K. Morgenstern, R. Berndt, W.-D. Schneider, Europhys. Lett. 2001, $56,254$.

[23] a) D. Andelman, P.-G. de Gennes, C. R. Acad. Sci. (Paris) 1988, 307, 233; b) D. Andelman, $J$. Am. Chem. Soc. 1989, 111, 6536.

[24] T. Yokoyama, S. Yokoyama, T. Kamikado, Y. Okuno, S. Mashiko, Nature 2001, 413, 619.

[25] N. Sato, K. Seki, H. Inokuchi, J. Chem. Soc., Faraday Trans. II 1981, 77, 1621.

[26] P. U. Biedermann, A. Levy, J. J. Stezowski, I. Agranat, Chirality 1995, 7, 199.

[27] Z. Zhi-lin J. Xue-yin, X. Shao-hong, T. Nagatomo and O. Omoto, J. Phys. D: Appl. Phys. 1998, 31, 32.
[28] B. Grünbaum, G. C. Shephard, 'Tilings and Patterns', Freeman, New York, 1987

[29] a) J. Stavans, Rep. Prog. Phys. 1993, 56, 733; b) B. Dubertret, T. Aste, H. M. Ohlenbusch, N. Rivier, Phys. Rev. E 1998, 58, 6368.

[30] F. Graner, Y. Jiang, E. Janiaud, C. Flament, Phys. Rev. E 2000, 63, 011402.

[31] G. Ungarn, X. Zeng, Soft Matter 2005, 1, 95.

[32] a) A. J. Stone, D.J. Wales, Chem. Phys. Lett. 1986, 128, 501; b) P. Simonis, C. Goffaux, P A. Thiry, L. P. Biro, P. Lambin, V. Meunier, Surf. Sci. 2002, 511, 319; c) A. Hashimoto, K. Suenaga, A. Gloter, K. Urita, S. Iijima, Nature 2004, 430, 870 .

[33] a) V. H. Crespi, L. X. Benedict, M. L. Cohen, S. G. Louie, Phys. Rev. B 1996, 53, 13303; b) M. Deza, P.W. Fowler, M. Shtogrin, K. Vietze, J. Chem. Inf. Comput. Sci. 2000, 40, 1325.

[34] G. R. Desiraju, Nature 2001, 412, 397.

[35] a) M. Tamres, J. Am. Chem. Soc. 1952, 74, 3375; b) M. Nishio, M. Hirota, Y. Umezawa, 'The $\mathrm{CH} / \pi$ interaction: evidence, nature, and consequences', Wiley, New York, 1998; c) U. Samanta, P. Chakrabarti, J. Chandrasekhar, J. Phys. Chem. A 1998, 102, 8964; d) S. Tsuzuki, K. Honda, T. Uchimaru, M. Mikami, K. Tanabe, J. Am. Chem. Soc. 2000, 122, 3746; e) M. Nishio, Cryst. Eng. Comm. 2004, 6, 230.

[36] R. D. Young, Physics Today November 1971, 42.

[37] J. K. Gimzewski, B. Reihl, J. H. Coombs, R. R. Schlittler, Z. Phys. B Cond. Matter 1988, 72, 497.

[38] X. H. Qiu, G. V. Nazin, W. Ho, Science 2003, 299, 542.

[39] Z.-C. Dong, X.-L.Guo, A. S. Trifonov, P. S. Dorozhkin, K. Miki, K. Kimura, S. Yokoyama, S. Mashiko, Phys. Rev. Lett. 2004, 92, 086801.

[40] R. Berndt, R. Gaisch, J. K. Gimzewski, B. Reihl, R. R. Schlittler, W.-D. Schneider, M. Tschudy, Science 1993, 262, 1425.

[41] Z.-C. Dong, A. Kar, P. Dorozhkin, K. Amemiya, T. Uchihashi, S. Yokoyama, T. Kamikado, S. Mashiko, T. Okamoto, Thin Solid Films 2003, 438-439, 262

[42] S. W. Wu, G. V. Nazin, X. Chen, X. H. Qiu, W. Ho, Phys. Rev. Lett. 2004, 93, 236802.

[43] W. C. Hung, C. D. Ho, C. P. Liu, Y. P. Lee, J. Phys. Chem. 1996, 100, 3927.
[44] A. Sassara, G. Zerza, M. Chergui, F. Negri, G. Orlandi, J. Chem. Phys. 1997, 107, 8731.

[45] Y. Wang, J. M. Holden, A. M. Rao, P. C. Eklund, U. D. Venkateswaran, D. L. Eastwood, R. L. Lideberg, G. Dresselhaus, M. S. Dresselhaus, Phys. Rev. B 1995, 51, 4547.

[46] V. Capozzi, G. Casamassima, G. F. Lorusso, A Minafra, R. Piccolo, T. Trovato, A. Valentini, Solid State Comm. 1996, 98, 853.

[47] W. Guss, J. Feldmann, E. O. Gobel, C. Taliani, H. Mohn, W. Muller, P. Haussler, H. U. Termeer, Phys. Rev. Lett. 1994, 72, 2644.

[48] D. J. van den Heuvel, I. Y. Chan, E. J. J. Groenen, M. Matsushita, J. Schmidt, G. Meijer, Chem. Phys. Lett. 1995, 233, 284.

[49] A. Sassara, G. Zerza, M. Chergui, J. Phys. Chem. 1998, 102, 3072.

[50] S. Kazaoui, N. Minami, Synth. Met. 1997, 86, 2345.

[51] M. Ichida, M. Sakai, T. Yajima, A. Nakamura, H. Shinohara, Chem. Phys. Lett. 1997, 271, 27.

[52] V. Capozzi, G. Perna, Thin Solid Films 2007, 515, 7247.

[53] B. Pettinger, 'Tip-Enhanced Raman Spectroscopy (TERS)', in 'Surface-Enhanced Raman Scattering - Physics and Applications', Top. Appl. Phys. 103, Eds. K. Kneipp, M. Moskovits, H. Kneipp, Springer, Berlin/ Heidelberg, 2006, pp. 217-242.

[54] I. Akimoto, M. Ashida, K. Kan'no, Chem. Phys. Lett. 1998, 292, 561.

[55] I. Akimoto, K. Kan'no, J. Phys. Soc. Jap. 2002, 71,630 .

[56] I. Akimoto, K. Kan'no, J. Lumin. 2000, 87-9, 788.

[57] J. H. Rice, J. P. Galaup, S. Leach, Chem. Phys. 2002, 279, 23.

[58] S. M. Argentine, K. T. Kotz, A. H. Francis, J. Am. Chem. Soc. 1995, 117, 11762.

[59] A. Kabakchiev, K. Kuhnke, T. Lutz, K. Kern, ChemPhysChem 2010, 11, 3412.

[60] F. Rossel, M. Pivetta, F. Patthey, E. Ćavar, A. P. Seitsonen, W.-D. Schneider, Phys. Rev. B 2011, 84, 075426.

[61] R. C. Powell, Z. G. Soos, J. Lumin. 1975, 11, 1

[62] I. Horcas, R. Fernandez, J. M. GomezRodriguez, J. Colchero, J. Gomez-Herrero, A. M. Baro, Rev. Sci. Instrum. 2007, 78, 013705.

[63] W. Gebauer, A. Langner, M. Schneider, M. Sokolowski, E. Umbach, Phys. Rev. B 2004, 69 , 155431. 